\title{
Nuclear Chiral EFT in the Precision Era
}

\author{
Evgeny Epelbaum* ${ }^{\dagger}$ \\ Institut für Theoretische Physik II, Ruhr-Universität Bochum, D-44780 Bochum, Germany \\ E-mail: evgeny. epelbaumerub. de
}

\begin{abstract}
Chiral effective field theory has established itself as the method of choice to study nuclear forces and low-energy nuclear dynamics. I review the status and prospects of this approach and discuss ongoing efforts to advance the precision frontier for $a b$ initio description of few-nucleon systems. Special emphasis is put on the precise determination of the two-nucleon force at fifth order in the chiral expansion, role of the chiral symmetry, the convergence pattern of the chiral expansion and the quantification of the theoretical uncertainties. The discussed topics are essential for ongoing studies towards elucidating the structure of the three-nucleon force which will be briefly addressed as well.
\end{abstract}

The 8th International Workshop on Chiral Dynamics, CD2015 ***

29 June 2015 - 03 July 2015

Pisa, Italy

\footnotetext{
${ }^{*}$ Speaker.

${ }^{\dagger}$ It is a great pleasure to thank my collaborators Hermann Krebs, Ulf-G. Meißner and all members of the LENPIC collaboration for sharing their insights into the discussed topics and the organizers of CD2015 for making this exciting workshop possible. I am also grateful to Hermann Krebs and Ulf-G. Meißner for useful suggestions on the manuscript. This work was supported in part by the ERC project 259218 NUCLEAREFT and by DFG (SFB/TR 16, "Subnuclear Structure of Matter").
} 


\section{Introduction}

The classical problem of the nuclear force has experienced a strong revival since the 1990s when Weinberg has shown that chiral perturbation theory (ChPT) can, in fact, be fruitfully applied beyond the Goldstone-boson and single-nucleon sectors [1,2]. While a direct application of ChPT to nucleon-nucleon (NN) scattering amplitude makes little sense due to the intrinsically non-perturbative nature of the problem, Weinberg has realized that all diagrams which violate the chiral power counting emerge from iterations of a Lippmann-Schwinger (LS) type of equation. The task of calculating the scattering amplitude thus naturally reduces to the conventional quantum mechanical $A$-body problem with nuclear potentials derived in ChPT. The resulting approach is commonly referred to as (nuclear) chiral effective field theory (EFT) and has the appealing features of being model-independent, systematically improvable and related to QCD via its symmetries, see Refs. [3, 4] for recent review articles.

Today, after a quarter of a century of intense research, nuclear chiral EFT is entering the precision era and is expected to shed light on the long-standing unsolved problems such as especially the three-nucleon force (3NF) problem [5,6]. This is becoming possible due to advances in the following three areas: First, the chiral expansion of the nuclear force has been/is being pushed to a sufficiently high order for making precision calculations. It is essential that the determination of an increasing number of unknown low-energy constants (LECs), which often plagues higher-order calculations in ChPT, is manageable in the nuclear sector thanks to the vast amount of neutronproton (np) and proton-proton (pp) scattering data and due to the fact that most of the unknown LECs contribute to the NN force. Secondly, chiral EFT provides a natural framework to quantify the theoretical uncertainties of the calculations, the issue which needs to be seriously addressed in order to make a meaningful comparison between theory and experiment. Finally, recent advances in ab initio few- and many-body methods including the coupled-cluster expansions [7], the no-core shell model [8], Green's function Monte Carlo method [9], the in-medium similarity renormalization group approach [10], and the self-consistent Green's functions technique [11] coupled with rapidly increasing computational recources open the exciting possibility of testing chiral EFT in nuclear structure and reaction calculations. A particularly promising new addition to the existing $a b$ initio methods is provided by Nuclear Lattice Effective Field Theory (NLEFT) [12], an approach which naturally combines chiral EFT with auxiliary-field quantum Monte Carlo lattice simulations to access light- and medium-mass nuclei, see Refs. [13, 14, 15, 16] for some most exciting results and the talk by Meißner for a review [17].

In this talk I will mainly focus on our recent work towards the development of the new generation of NN potentials up to fifth order in the chiral expansion $[18,19]$ along with the novel approach for error analysis. I will also discuss selected applications beyond the NN system. This paper is organized as follows. In section 2, I will discuss the foundations of nuclear chiral EFT and focus on its application to the NN system. Section 3 addresses the issue of estimating the theoretical unsertainties due to the truncation of the chiral expansion. Selected applications to the three-nucleon system are presented in section 4. Finally, section 5 gives the outlook on ongoing and future research in this field. 


\section{A new generation of chiral NN forces up to fifth order}

\subsection{Foundations}

The starting point in the derivation of the nuclear forces and currents is the most general chiral invariant effective Langangian for pions and nucleons. Given the non-relativistic nature of the problem at hand and the desire to treat nuclear dynamics in the framework of the quantum mechanical $A$-body Schrödinger equation, it is natural to utilize the heavy-baryon approach for the description of the nucleon fields. For the explicit form of the $\pi N$ heavy-baryon Lagrangian up to fourth order, sufficient for the applications discussed in this paper, see Ref. [20]. The choice of pions and nucleons as explicit degrees of freedom in the Lagrangian is dictated by the energy range the resulting EFT is supposed to be applicable to. As an alternative, for few-nucleon reactions at very low energies with typical three-momenta of the nucleons $|\vec{p}|$ being well below the pion mass $M_{\pi}$ and/or for matching to lattice QCD results at unphysically heavy quark masses [21], the socalled pion-less EFT formulation is expected to provide a more efficient approach, see the talk by Schindler for the application of this method to parity-violating few-nucleon reactions [22]. On the other hand, the explicit inclusion of the $\Delta(1232)$ resonance in the effective chiral Lagrangian along the lines of Ref. [23] is expected to improve the convergence pattern of the chiral expansion and to increase its applicability range to higher energies. Here and in what follows, I will focus on the standard formulation of chiral EFT based on pions and nucleons as the only degrees of freedom.

The effective chiral Lagrangian represents an infinite set of terms which respect the approximate spontaneously broken chiral symmetry of QCD. They can be classified by the number of derivatives and/or pion mass insertions. For applications in the few-nucleon sector, it is convenient to assign the chiral dimension $\Delta_{i}$ to a vertex $i$ which is defined as [1,2]

$$
\Delta_{i}=d_{i}+\frac{1}{2} n_{i}-2
$$

where $d_{i}$ is the number of derivatives or $M_{\pi}$-insertions while $n_{i}$ refers to the number of nucleon field operators. Using dimensional counting, it is straightforward to show that a connected diagram with $N$ nucleons and $L$ loops constructed out of $V_{i}$ vertices of type $\Delta_{i}$ scales as $Q^{v}$ with the chiral dimension $v$ given by

$$
v=-4+2 N+2 L+\sum_{i} V_{i} \Delta_{i}
$$

Here, $Q \in\left\{M_{\pi} / \Lambda_{b},|\vec{p}| / \Lambda_{b}\right\}$ denotes the expansion parameter of chiral EFT with $\Lambda_{b}$ being the breakdown scale to be specified below. The above expression has a slightly different form than the one derived originally by Weinberg in Ref. [1] as discussed in Ref. [24]. Notice that since the effective Lagrangian involves only non-renormalizable interactions, the condition $\Delta_{i} \geq 0$ holds true for all $i$. This implies that $v$ is bounded from below and that at each fixed order, a finite number of diagrams made out of a finite number of vertices can contribute.

Since Eq. (2.2) is based solely on dimensional arguments, it does not take into account the enhancement of reducible ladder-type diagrams involving purely nucleonic intermediate states due to the appearance of pinch singularities (in the static limit) when performing integrals over zeroth components of the loop momenta. Clearly, such infrared singularities are regularized by the nucleon kinetic energy term which has to be explicitly kept in the corresponding propagators. Still, 


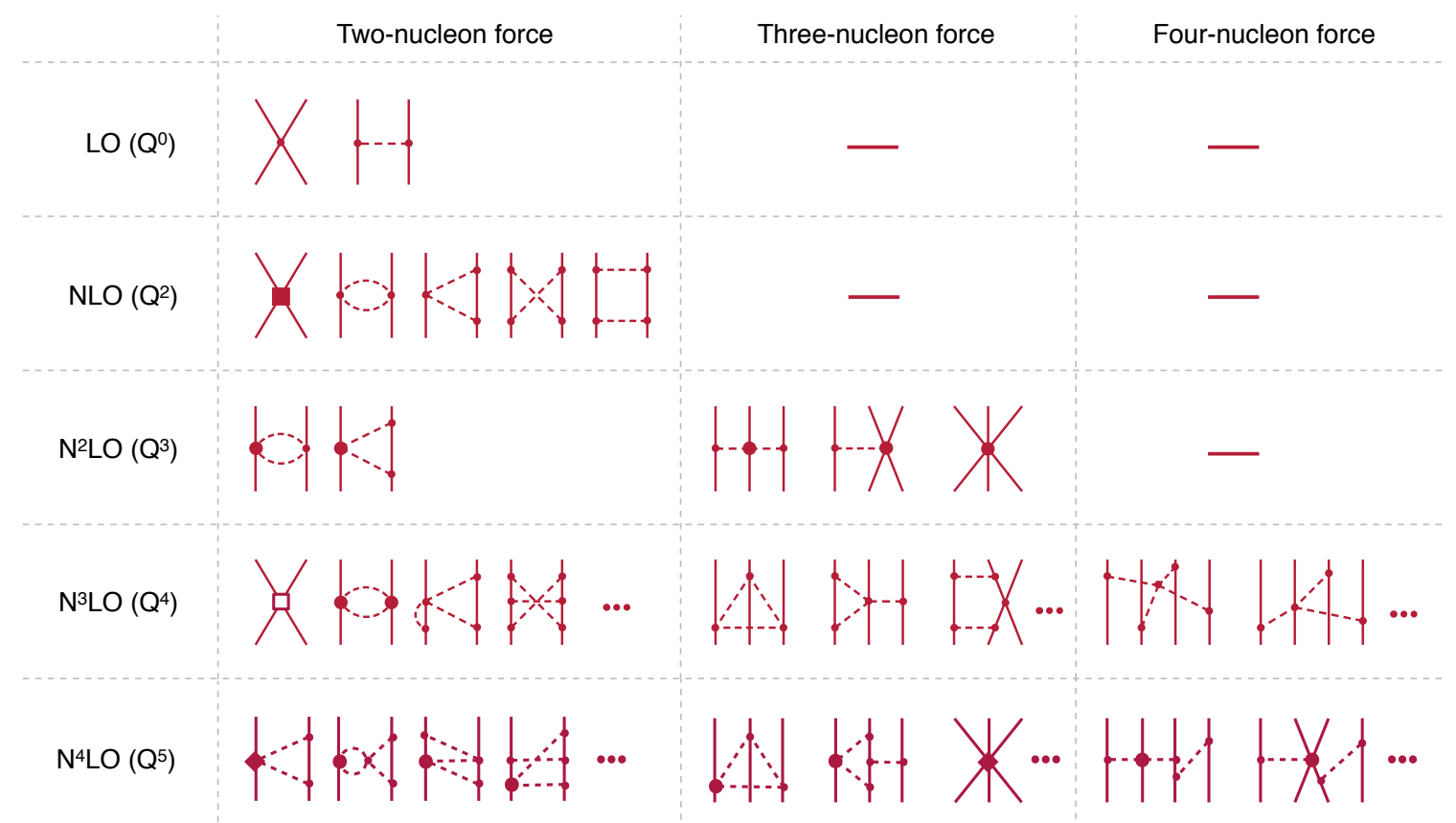

Figure 1: Chiral expansion of the nuclear forces. Solid and dashed lines refer to nucleons and pions, respectively. Solid dots, filled circles, filled rectangles, filled diamonds and open rectangles refer to the vertices of dimension $\Delta_{i}=0, \Delta_{i}=1, \Delta_{i}=2, \Delta_{i}=3$ and $\Delta_{i}=4$, respectively.

the resulting contributions to the amplitude are enhanced by powers of $m_{N} /|\vec{p}|$, where $m_{N}$ refers to the nucleon mass, as compared to estimates based on dimensional analysis and underlying the derivation of Eq. (2.2). Fortunately, the contributions of the enhanced ladder-like diagrams can be easily and efficiently resummed by solving the LS integral equation (or its generalizations in the case of three- and more-nucleon systems) whose kernel involves all possible irreducible graphs which obey the scaling according to Eq. (2.2) and are derivable in perturbation theory. This is the essence of what is commonly referred to as Weinberg's approach to nuclear chiral EFT. The set of all possible irreducible contributions to the scattering amplitude can be viewed as the interaction part of the nuclear Hamiltonian and comprises two-, three- and more-nucleon forces. The approach outlined above is straightforwardly generalizable to reactions involving external sources and allows one to derive exchange currents consistent with the nuclear forces.

It is a simple exercise to enumerate the various diagrams which may contribute to the nuclear force at a given order $v$ by looking at Feynman rules for the chiral Lagrangian and applying Eq. (2.2), see Fig. 1. Here, it is understood that the shown diagrams only serve the purpose of visualization of the corresponding contributions and do not have the meaning of Feynman graphs. In particular, one needs to separate out the irreducible pieces in order to avoid double counting. Notice further that while one can draw three-nucleon diagrams at next-to-leading order (NLO), the resulting contributions are either reducible or suppressed by one power of $Q / m_{N}$ [25]. As an immediate consequence of the chiral power counting in Eq. (2.2), one observes the suppression of many-body forces [26], the feature, that has always been assumed but could be justified only in the context of chiral EFT. 
So far, I have not yet discussed the important issue of identifying the irreducible parts of the scattering amplitude, i.e. the actual derivation of the nuclear forces. This can be achieved using a veriety of approaches. In particular, old-fashioned time-ordered perturbation theory was employed by Weinberg in his original papers $[2,25]$ and by Ordonez et al. in their first numerical analysis of NN scattering [27]. More recently, this method was taken over by the Jlab-Pisa group to derive electromagnetic [28, 29, 30] and weak [31] exchange currents, see the talk by Schiavilla [32] for more details. We used another well-known technique commonly referred to as the method of unitary transformation (UT) [33, 34]. The main idea of this approach consists in block-diagonalization of the pion-nucleon Hamiltonian in Fock space via a suitable chosen UT. The corresponding unitary operator can be determined perturbatively employing the chiral power counting as explained in detail in Refs. [35, 36, 37, 24], see also Ref. [38] for a closely related approach. The method of UT can be straightforwardly applied to derive the exchange currents [39, 40]. Finally, nuclear forces can also be defined by calculating Feynman diagrams and matching the results to the LS equation, see Ref. [41] for more detail.

Irregardless of the employed approach to derive nuclear potentials, it is important to keep in mind that, in contrast to S-matrix elements, nuclear forces and exchange currents are not uniquely defined and can always be changed by unitary transformations or, equivalently, by changing the basis in the Fock space. The intrinsic unitary ambiguity of the Hamiltonian and exchange currents turns out to play the crucial role in maintaining their renormalizability. More precisely, these quantities will generally not stay finite after evaluating the loop integrals and replacing the bare LECs with the renormalized ones. This is in a strong contrast with on-shell scattering amplitudes calculated in ChPT, for which all ultraviolet divergences do, of course, cancel against the corresponding counterterms. This issue was first raised in Ref. [42], where renormalizability of the Hamiltonian could finally be established for a specific choice of the unitary transformations (i.e. for a particular choice of basis in the Fock space). We also followed the same approach of systematically exploiting the freedom in the choice of the basis in the Fock space to maintain renormalizability of the electromagnetic exchange charge and current operators to leading one-loop order in Refs. [39, 40]. This is in contrast with the results of the Jlab-Pisa group [28, 29, 30, 31], whose expressions for the one-pion exchange contributions contain ultraviolet divergences which are not cancelled by counterterms from the effective chiral Lagrangian.

Having outlined the various techniques to derive the nuclear Hamiltonian, I now briefly discuss the current state of the art for the contributions shown in Fig. 1. The NN force up to next-to-nextto-leading order $\left(\mathrm{N}^{2} \mathrm{LO}\right)$ has been known since about two decades and was derived using all three approaches mentioned above [27, 41,35]. The order- $Q^{4}\left(\mathrm{~N}^{3} \mathrm{LO}\right)$ corrections to the NN potential have been worked out in a series of papers by Kaiser [43, 44, 45, 46] about fifteen years ago using an S-matrix based approach and employing Cutkosky's cutting rules to simplify the calculation of loop integrals. These results provided a basis for the development of the first generation of chiral $\mathrm{NN}$ potentials at $\mathrm{N}^{3} \mathrm{LO}[47,48]$. Very recently, the same technique was used by Entem et al. [49] to work out the order- $Q^{5}\left(\mathrm{~N}^{4} \mathrm{LO}\right)$ corrections to the two-pion exchange $\mathrm{NN}$ potential ${ }^{1}$ and even the dominant order- $Q^{6}\left(\mathrm{~N}^{5} \mathrm{LO}\right)$ terms [50]. Notice that the leading $3 \pi$-exchange potentials at $\mathrm{N}^{3} \mathrm{LO}$ turn out to be negligibly weak [43,44] and were not included explicitly in Ref. [48]. While the

\footnotetext{
${ }^{1}$ We have independently calculated these contributions [19] and have verified the results obtained by Entem et al..
} 
subleading $3 \pi$-exchange potentials at $\mathrm{N}^{4} \mathrm{LO}$ are stronger in magnitude, their short-range nature permits their implicit representation via contact interactions [19].

The expressions for the leading $3 \mathrm{NF}$ at $\mathrm{N}^{2} \mathrm{LO}$ resulting from tree-level diagrams shown in Fig. 1 have been known since a long time $[26,51]$ and depend on two LECs $c_{D}$ and $c_{E}$ which accompany the contact interactions in the second and third diagram, respectively. The first corrections at $\mathrm{N}^{3} \mathrm{LO}$ encompass numerous one-loop diagrams. Their calculation has been accomplished in Refs. [52, 53, 54]. Interestingly, the resulting contributions do not involve any unknown LECs. The long- and intermediate-range corrections to the $3 \mathrm{NF}$ at $\mathrm{N}^{4} \mathrm{LO}$ have been derived recently using the method of unitary transformation $[55,56]$ while the purely contact $3 \mathrm{NF}$ terms have been worked out in Ref. [57] and involve 10 unknown LECs. The derivation of the remaining contributions involving NN contact interactions is in progress [58]. Finally, the leading four-nucleon force at $\mathrm{N}^{3} \mathrm{LO}$ has been calculated in Refs. $[42,24]$ and is also parameter-free. The corrections at $\mathrm{N}^{4} \mathrm{LO}$ have not been studied yet.

\subsection{Renormalization of the Schrödinger equation and regularization of the potential}

The chiral nuclear potentials discussed in the previous section are constructed to be used in the $A$-body Schrödinger equation

$$
\left[\left(\sum_{i=1}^{A} \frac{-\Delta_{i}}{2 m_{N}}+\mathscr{O}\left(m_{N}^{-3}\right)\right)+V_{2 N}+V_{3 N}+V_{4 N}+\ldots\right]|\Psi\rangle=E|\Psi\rangle
$$

where the nuclear forces $V_{2 N}, V_{3 N}, V_{4 N}, \ldots$, are derived by means of the chiral expansion as discussed above. Consider, for the sake of simplicity, the NN system where the Schrödinger equation can be conveniently cast into the LS integral equation for the $T$-matrix. In the operator form, this equation reads

$$
\hat{T}=\hat{V}_{N N}+\hat{V}_{N N} \hat{G}_{0} \hat{T}=\hat{V}_{N N}+\hat{V}_{N N} \hat{G}_{0} \hat{V}_{N N}+\hat{V}_{N N} \hat{G}_{0} \hat{V}_{N N} \hat{G}_{0} \hat{V}_{N N}+\ldots
$$

where $\hat{G}_{0}$ is the free resolvent operator. Iterations of the NN potential in the LS equation generate ultraviolet (UV) divergences which are cancelled by counterterms of the contact interaction type. While this cancellation certainly holds true for the potential calculated up to an infinite order in the chiral expansion which involves all possible counterterms compatible with the symmetries, it is not ensured anymore if a truncated expression for $V_{N N}$ is employed. Indeed, it is easy to verify that the LS equation for the leading-order (LO) one-pion exchange potential (OPEP) is linearly divergent. Consequently, its iterations in any given spin-triplet partial wave generate UV divergences whose cancellation requires the introduction of infinitely many counterterms with increasing powers of momenta and/or $M_{\pi}$.

The simplest way to circumvent this problem is by treating the OPEP perturbatively [59]. The resulting EFT approach, however, fails (at least) in certain spin-triplet channels due to the lack of convergence [60]. An alternative solution has been proposed recently in Ref. [61], where it was pointed out that the linearly divergent UV behavior of the LS equation for the OPEP can be traced back to the non-relativistic expansion being carried out prior to solving the dynamical equation. Using, instead of the LS equation, a three-dimensional integral equation which maintains relativistic elastic unitarity such as e.g. the Kadyshevsky equation [62] results in a milder UV 
divergence of a logarithmic type. The resulting LO equation for the OPEP and derivative-less contact interactions is renormalizable in the sense that all UV divergences generated by iterations can be absorbed into the two LO derivative-less contact interactions. Clearly, the intrinsically non-relativistic nature of the problem is not affected by this procedure, and it is perfectly fine to perform, if desired, a $1 / m_{N}$-expansion of the amplitude after solving the integral equation [61]. This renormalizable approach outlined above permits a complete removal of the UV cutoff and has been applied at LO to chiral extrapolations [63] and to the electromagnetic form factors of the deuteron [64]. Higher-order corrections have to be included perturbatively in order to maintain renormalizability. ${ }^{2}$

While the approach outlined above is the only way to completely remove the UV cutoff in calculations with the OPEP being treated non-perturbatively within the framework of an EFT I am aware of ${ }^{3}$, its extention to higher orders and/or heavier systems represents a challenging task. An alternative and much simpler (but still valid from the EFT point of view) approach is to introduce a finite UV regulator which is to be chosen of the order of the relevant hard scale in the problem as advocated in Refs. [70, 71]. As already mentioned, taking the values of the UV cutoff $\Lambda$ in the LS equation well beyond the pertinent hard scale may result in the breakdown of low-energy theorems [69] which signals the violation of basic principles of EFT. I refer interested readers to our work [69], which provides an explicit example of such a failure and brings further analytical insights into these topics by considering an exactly solvable model. The finite-regulator scheme is very well suited for applications using $a b$ initio few- and many-body methods and has been adopted in the most sophisticated nuclear chiral EFT calculations carried out up to date. This is the approach I will focus on in the following.

For calculations with a finite UV cutoff, it is important to employ such a functional form of the regulator which avoids introducing unnecessary artifacts. In the new generation of chiral potentials of Refs. [18, 19], we used a coordinate-space regularization procedure for the long-range components $V_{\text {long }}(\vec{r})$,

$$
V_{\text {long }}(\vec{r}) \rightarrow V_{\text {long }}^{\text {Reg. }}(\vec{r})=V_{\text {long }}(\vec{r}) f\left(\frac{r}{R}\right) \quad \text { with } \quad f(x)=\left(1-\exp \left(-x^{2}\right)\right)^{6},
$$

where the cutoff distance $R$ is chosen in the range of $R=0.8 \ldots 1.2 \mathrm{fm}$ in agreement with the expected breakdown distance of $\sim 0.8 \mathrm{fm}$ of the chiral expansion for the pion-exchange potential [72]. A similar regulator was employed in Refs. $[73,74]$. In momentum space, the regularization takes the form

$$
V_{\text {long }}(\vec{q}) \rightarrow V_{\text {long }}^{\text {reg }}(\vec{q})=V_{\text {long }}(\vec{q})-\int \frac{d^{3} l}{(2 \pi)^{3}} V_{\text {long }}(\vec{l}) \mathrm{FT}_{\vec{q}-\vec{l}}[1-f]
$$

where FT stays for the Fourier-Transform and $\vec{q}$ is the momentum transfer. Given that FT $[1-f]$ is a short-range operator, it is clear that the regulator does, per construction, not affect the long-range

\footnotetext{
${ }^{2}$ Higher-order contact interactions can also be treated non-perturbatively, see Refs. [65] for more details.

${ }^{3}$ Several authors have explored the possibility of removing the UV regulator in the context of the LS equation via performing self-adjoint extentions of singular potentials due to one- (and more-) pion exchanges, see e.g. [66, 67, 68]. Such manifestly non-perturbative approaches have been demonstrated in Ref. [69] to be, in general, incompatible with the principles of EFT in spite of being able to yield finite results for the scattering amplitude. It is important to remember that the UV regulator can be removed from the calculations only after all UV divergences have been subtracted.
} 


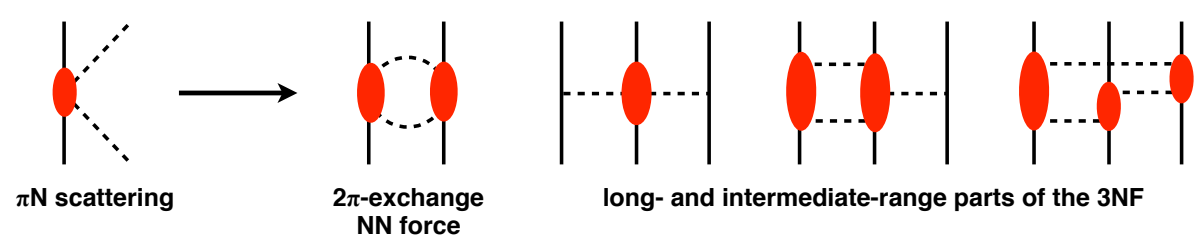

Figure 2: The long-range part of the nuclear force is completely predicted by the chiral symmetry of QCD and experimental information on the pion-nucleon system.

part of the interaction and thus maintains the analytic structure of the amplitude in the low-energy domain. This feature is in contrast with the non-local momentum-space regulator employed in the first-generation NN potentials of Refs. $[47,48]$ of the type

$$
V\left(\vec{p}, \vec{p}^{\prime}\right) \rightarrow V^{\mathrm{reg}}\left(\vec{p}, \vec{p}^{\prime}\right)=V\left(\vec{p}, \vec{p}^{\prime}\right) \exp \left(-\frac{p^{2 n}+p^{2 n}}{\Lambda^{2 n}}\right), \quad n=2,3,
$$

where $\vec{p}, \vec{p}^{\prime}$ are the initial and final momenta of the nucleons in the center of mass system (CMS), which distorts the long-range part of the interaction. Another advantage of the regulator in Eq. (2.5) is that it cuts off precisely the undesired short-range components of the pion exchange contributions which cannot be meaningfully predicted in chiral EFT instead of their large-momentum parts as does the non-local regulator in Eq. (2.7). This makes the additional spectral-function regularization (SFR) [75] of the two-pion exchange components, which was used e.g. in Refs. [48, 76] to tame the unphysically strong attraction at short distances at $\mathrm{N}^{2} \mathrm{LO}$ [41], obsolete. This is a particularly welcome feature in view of the ongoing and upcoming 3NF studies, in which the implementation of the SFR would be rather non-trivial. The insensitivity of the calculated NN observables to the value of the exponent in Eq. (2.5) is demonstrated in [18]. For contact interactions, we used in Refs. $[18,19]$ a non-local Gaussian regulator in momentum space with the cutoff set to $\Lambda=2 / R$.

\subsection{Determination of the LECs}

I am now in the position to specify the employed values of the various LECs and begin with the long-range part of the potential due to exchange of pion(s). Here, the framework of chiral EFT shows its full power by allowing one to predict the long-range part of the nuclear force in a parameter-free way using the available experimental information on the pion-nucleon system and exploiting the constraints due to the chiral symmetry of QCD as visualized schematically in Fig. 2. At orders $\mathrm{N}^{2} \mathrm{LO}, \mathrm{N}^{3} \mathrm{LO}$ and $\mathrm{N}^{4} \mathrm{LO}$, one needs to specify the values of the order- $Q^{2}$, order- $Q^{3}$ and order- $Q^{4} \pi N$ LECs $c_{i}, d_{i}$ and $e_{i}$, respectively. At $\mathrm{N}^{2} \mathrm{LO}$ and $\mathrm{N}^{3} \mathrm{LO}$, we used in [18] the values of $c_{1}=-0.81, c_{2}=3.28, c_{3}=-4.69, c_{4}=3.40, \bar{d}_{1}+\bar{d}_{2}=3.06, \bar{d}_{3}=-3.27, \bar{d}_{5}=0.45$ and $\bar{d}_{14}-\bar{d}_{15}=-5.65$ from the order- $Q^{3}$ fits to $\pi N$ data in the physical region [77] and inside the Mandelstam triangle [78]. Further, the LEC $d_{18}$ is adjusted to reproduce the observed value of the Goldberger-Treiman discrepancy. Here and in the following, the values of the LECs are given in units of $\mathrm{GeV}^{-n}$. The bars over the LECs indicate that I am using the convention of Ref. [77] by setting the dimensional regularization scale equal to the pion mass. At $\mathrm{N}^{4} \mathrm{LO}$, we employ the values from our order- $Q^{4}$ fit to Karlsruhe-Helsinki partial-wave analysis of $\pi N$ scattering [55], namely: $c_{1}=-0.75, c_{2}=3.49, c_{3}=-4.77, c_{4}=3.34, \bar{d}_{1}+\bar{d}_{2}=6.21, \bar{d}_{3}=-6.83, \bar{d}_{5}=0.78, \bar{d}_{14}-\bar{d}_{15}=$ 
$-12.02, \bar{e}_{14}=1.52$ and $\bar{e}_{17}=-0.37$. These values are in a reasonable agreement with the ones of Ref. [79] from a $Q^{4}$ fit to $\pi N$ scattering data and with the ones of Ref. [80] from matching to the subthreshold coefficients obtained from the Roy-Steiner analysis of $\pi N$ scattering [81]. Still, the differences between the values found in these determinations indicate sizable uncertainties in the LECs, whose impact on the nuclear forces needs to be addressed in future studies.

I now turn to the short-range part of the potential. Adopting the standard power counting rules for short-range operators, which are based on naive dimensional analysis ${ }^{4}$, one has to take into account (in the isospin limit) 2 order- $Q^{0}$ contact terms at LO, 7 additional order- $Q^{2}$ contact interactions at $\mathrm{NLO}$ and $\mathrm{N}^{2} \mathrm{LO}$ and 15 additional order- $Q^{4}$ contact terms at $\mathrm{N}^{3} \mathrm{LO}$ and $\mathrm{N}^{4} \mathrm{LO}$. This leaves one with 24 unknown LECs in total, which have been determined from a fit to np and pp S-, $\mathrm{P}$ - and D-waves and to the mixing angles $\varepsilon_{1}$ and $\varepsilon_{2}$ of the Nijmegen partial wave analysis (NPWA) [84] for five different choices of the regulator $R$

$$
\left\{R_{1}, R_{2}, R_{3}, R_{4}, R_{5}\right\}=\{0.8 \mathrm{fm}, 0.9 \mathrm{fm}, 1.0 \mathrm{fm}, 1.1 \mathrm{fm}, 1.2 \mathrm{fm}\} .
$$

We also included 2 (3) isospin-violating contact interactions up to $\mathrm{N}^{3} \mathrm{LO}\left(\right.$ at $\left.\mathrm{N}^{4} \mathrm{LO}\right)$ which account for isospin-breaking effects in the ${ }^{1} \mathrm{~S}_{0}$ partial wave. A detailed description of the fit procedure and the treatment of relativistic and isospin-breaking terms can be found in the original papers $[18,19]$. Here, we only emphasize that all LECs were found to be of a natural size for all five choices of the cutoff $R$, see Table II of Ref. [18]. ${ }^{5}$

\subsection{Results for phase shifts}

The resulting np phase shifts are plotted in Fig. 3 as functions of the laboratory energies in comparison with the NPWA and the single-energy partial wave analysis of Ref. [85] for the cutoff choice of $R=0.9 \mathrm{fm}$. In all cases, one observes a good and natural convergence pattern with the results at $\mathrm{N}^{4} \mathrm{LO}$ being almost indistinguishable from those at $\mathrm{N}^{3} \mathrm{LO}$.

To get more quantitative insights, it is instructive to look at $\chi^{2}$ per datum for the description of the Nijmegen np and pp phase shifts. For a phase shift or mixing angle $\delta$ in a channel $X$ at a given energy, we assign the error of the NPWA to be

$$
\Delta_{X}=\max \left(\Delta_{X}^{\mathrm{NPWA}},\left|\delta_{X}^{\mathrm{NijmI}}-\delta_{X}^{\mathrm{NPWA}}\right|,\left|\delta_{X}^{\mathrm{NijmII}}-\delta_{X}^{\mathrm{NPWA}}\right|,\left|\delta_{X}^{\mathrm{Reid} 93}-\delta_{X}^{\mathrm{NPWA}}\right|\right),
$$

where $\Delta_{X}^{\mathrm{NPWA}}$ is the statistical error of the NPWA [84], while $\delta_{X}^{\mathrm{NijmI}}, \delta_{X}^{\mathrm{NijmI}}$ and $\delta_{X}^{\mathrm{Reid93}}$ refer to the results based on the Nijmegen I, II and Reid93 NN potentials of Ref. [86] which provide a nearly optimal description of the same database as employed in the NPWA and can be regarded as alternative PWA. I emphasize, however, that the resulting $\chi^{2}$ per datum does not allow for a simple statistical interpretation due to the artificially chosen errors and, in particular, can take values $<1$ without indicating any inconsistencies. Therefore, in order to avoid a possible confusion or misinterpretation, I will use the notation $\tilde{\chi}^{2}$.

In table 1 , I give $\tilde{\chi}^{2}$ per datum for the description of the np and pp phase shifts of the NPWA at the energies of $E_{\text {lab }}=1,5,10,25,50,100,150$ and $200 \mathrm{MeV}$. As expected, one observes the

\footnotetext{
${ }^{4}$ For alternative suggestions see e.g. Refs. [66, 82, 83].

${ }^{5}$ The LECs accompanying the order- $Q^{4} \mathrm{~S}$-wave contact interactions, however, turn out to be significantly larger in magnitude than the other LECs.
} 

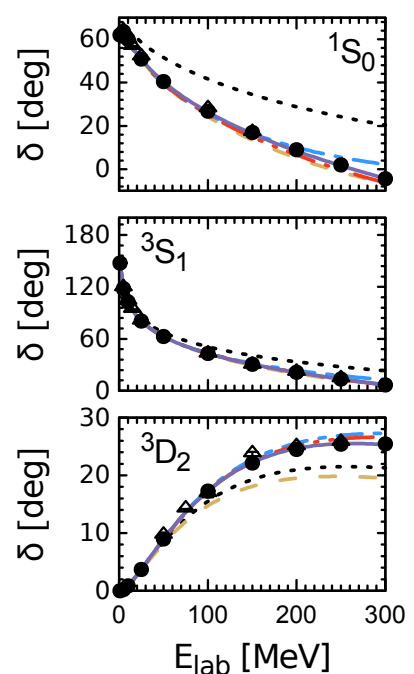
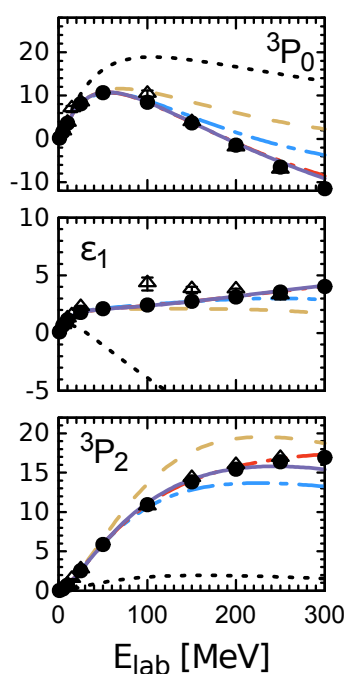
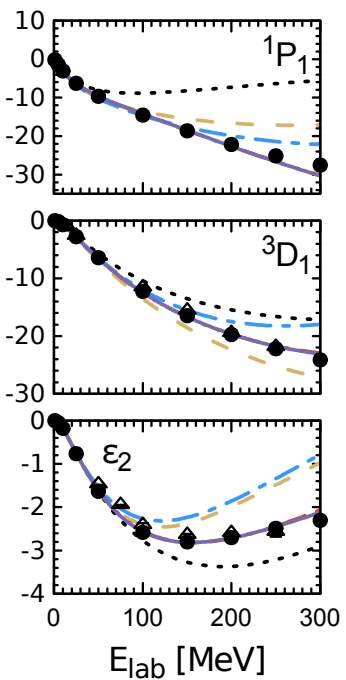
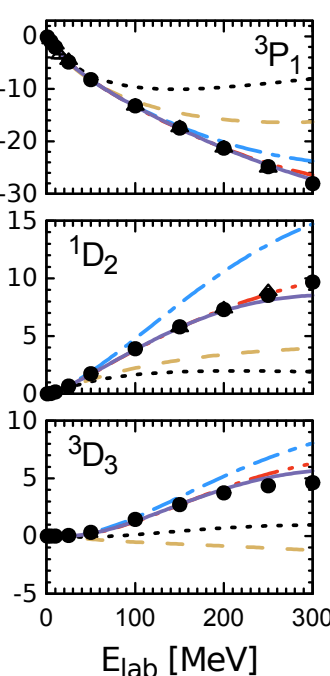

Figure 3: Chiral expansion of the np phase shifts for the cutoff $R=0.9 \mathrm{fm}$ in comparison with NPWA [84] (solid dots) and the GWU single-energy np partial wave analysis [85] (open triangles). Dotted (black), dashed (brown), dash-dotted (blue), dash-double-dotted (red) and solid (violet) lines show the results at $\mathrm{LO}, \mathrm{NLO}, \mathrm{N}^{2} \mathrm{LO}, \mathrm{N}^{3} \mathrm{LO}$ and $\mathrm{N}^{4} \mathrm{LO}$, respectively.

Table 1: $\tilde{\chi}^{2} /$ datum for the description of the Nijmegen np and pp phase shifts [84] for the cutoff $R=0.9 \mathrm{fm}$. The numbers in the round brackets give the number of adjustable isospin-invariant contact interactions at the corresponding order with the subscripts referring to the number of isospinbreaking contact terms. Only those channels are included which have been used in the $\mathrm{N}^{3} \mathrm{LO} / \mathrm{N}^{4} \mathrm{LO}$ fits, namely the $\mathrm{S}$-, $\mathrm{P}$ - and $\mathrm{D}$-waves and the mixing angles $\varepsilon_{1}$ and $\varepsilon_{2}$.

\begin{tabular}{lccccc}
\hline$E_{\text {lab bin }}$ & $\mathrm{LO}\left(2_{[2]}\right)$ & $\mathrm{NLO}\left(+7_{[0]}\right)$ & $\mathrm{N}^{2} \mathrm{LO}\left(+0_{[0]}\right)$ & $\mathrm{N}^{3} \mathrm{LO}\left(+15_{[0]}\right)$ & $\mathrm{N}^{4} \mathrm{LO}\left(+0_{[1]}\right)$ \\
\hline \multicolumn{2}{l}{ neutron-proton phase shifts } & & & & 0.7 \\
$0-100$ & 360 & 31 & 4.5 & 0.7 & 0.3 \\
$0-200$ & 480 & 63 & 21 & 0.8 & 0.3 \\
\hline \multicolumn{2}{l}{ proton-proton phase shifts } \\
$0-100$ & 5750 & 102 & 15 & 0.7 & 0.6 \\
$0-200$ & 9150 & 560 & 130 & 0.7 \\
\hline
\end{tabular}

improved description of the phase shifts with increasing chiral order. These results constitute an important consistency check of the theoretical approach and provide a beautiful illustration of its predictive power. In particular, one observes a strong decrease in the value of $\tilde{\chi}^{2}$ per datum at $\mathrm{N}^{2} \mathrm{LO}$ which is solely due to inclusion of the parameter-free order- $Q^{3}$ TPE. Similarly, we find a significant decrease in $\tilde{\chi}^{2}$ per datum at $\mathrm{N}^{4} \mathrm{LO}$ which, for the pp case, again emerges entirely from the predicted parameter-free order- $Q^{5}$ contributions to the TPE potential. The obtained results suggest - fully in line with the Weinberg power counting [1] - that the theoretical uncertainty at NLO and $\mathrm{N}^{3} \mathrm{LO}$ is dominated by the neglected TPE contributions at orders $Q^{3}$ and $Q^{5}$, respectively. Indeed, if certain order- $Q^{4}$ and order- $Q^{6}$ contact interactions would have to be promoted to lower orders in violation 

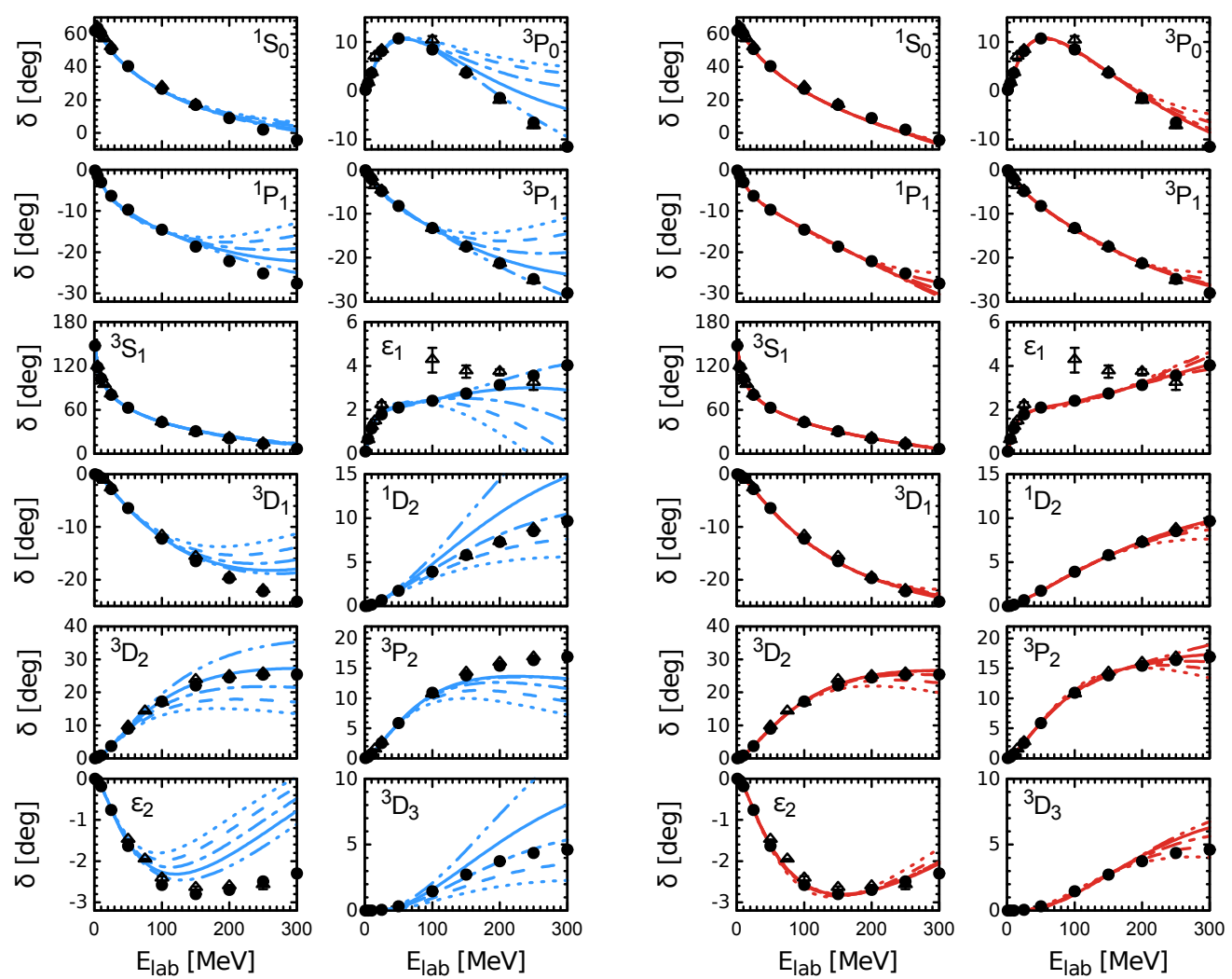

Figure 4: Cutoff dependence of the phase shifts calculated at $\mathrm{N}^{2} \mathrm{LO}$ (left panel) and $\mathrm{N}^{3} \mathrm{LO}$ (right panel). Dotted, dashed, dashed-dotted, solid and dashed-double-dotted lines show the results for $R=1.2 \mathrm{fm}, 1.1 \mathrm{fm}, 1.0 \mathrm{fm}, 0.9 \mathrm{fm}$ and $0.8 \mathrm{fm}$, respectively. For remaining notation see Fig. 3 .

with naive dimensional analysis as suggested e.g. in [66, 83, 82], the inclusion of the order- $Q^{3}$ and order- $Q^{5}$ TPE contributions alone would not result in the improved accuracy of the fits at $\mathrm{N}^{2} \mathrm{LO}$ and $\mathrm{N}^{4} \mathrm{LO}$.

I now briefly address the residual cutoff dependence of our results. Fig. 4 shows the np phase shifts at $\mathrm{N}^{2} \mathrm{LO}$ and $\mathrm{N}^{3} \mathrm{LO}$ for all considered choices of the regulator. As expected, the residual cutoff dependence at $\mathrm{N}^{2} \mathrm{LO}$ is efficiently absorbed into redefinition of the order- $Q^{4}$ contact interactions at $\mathrm{N}^{3} \mathrm{LO}$. I do not show the results at $\mathrm{N}^{4} \mathrm{LO}$, but they turn out to be very similar to those at $\mathrm{N}^{3} \mathrm{LO}$ what concerns the dependence on the regulator $R$.

It is also instructive to look at $\tilde{\chi}^{2}$ per datum for the reproduction of the phase shifts of the NPWA as a function of the cutoff $R$. Here, for the sake of brevity, I restrict myself to $\mathrm{N}^{3} \mathrm{LO}$ and to the single energy bin of $E_{\text {lab }}=0-200 \mathrm{MeV}$. We find the following pattern for np phase shifts by decreasing the values of the regulator starting from the softest choice of $R=1.2 \mathrm{fm}$ :

$$
\tilde{\chi}^{2} / \text { datum }=1.8_{R=1.2 \mathrm{fm}} \rightarrow 0.8_{R=1.1 \mathrm{fm}} \rightarrow 0.6_{R=1.0 \mathrm{fm}} \rightarrow 0.7_{R=0.9 \mathrm{fm}} \rightarrow 0.8_{R=0.8 \mathrm{fm}},
$$

while the results for pp channels are:

$$
\tilde{\chi}^{2} / \text { datum }=8.2_{R=1.2 \mathrm{fm}} \rightarrow 2.2_{R=1.1 \mathrm{fm}} \rightarrow 0.6_{R=1.0 \mathrm{fm}} \rightarrow 0.7_{R=0.9 \mathrm{fm}} \rightarrow 2.1_{R=0.8 \mathrm{fm}} .
$$


As expected, the softest choice of the regulator of $R=1.2 \mathrm{fm}$ leads to the worst quality of the fit indicative of the largest amount of cutoff artifacts. Decreasing the value of $R$ improves the description of phase shifts. The improvement stops around $R=0.9-1.0 \mathrm{fm}$, and the fits start deteriorating for $R=0.8 \mathrm{fm}$. This is exactly the pattern one expects to observe in calculations within this theoretical framework as discussed in section 2.2 and in Refs. [70, 71, 69].

\section{Theoretical uncertainty}

Uncertainty quantification is a key for performing a meaningful comparison between theoretical predictions/postdictions and experimental data [87]. The various sources of uncertainty in our calculations include: (i) uncertainty in the knowledge of $\pi N$ LECs which determine the long-range part of the interaction, (ii) systematic and statistical uncertainty in the determination of NN contact interactions, (iii) uncertainty in the NPWA used to determine the LECs and (iv) systematic uncertainty due to truncation of the chiral expansion. The impact of the statistical uncertainty of the LECs accompanying contact interactions on $\mathrm{np}$ and pp phase shifts was quantified in Ref. [88] at $\mathrm{N}^{2} \mathrm{LO}$ and found to be negligible at the accuracy level of our calculations. Similarly, we believe that the systematic uncertainty of the NPWA used as input in our analysis has a minor effect on our results, but this needs to be explicitly verified by using real data in the fits. Here I will focus on quantifying the effects of the truncated higher-order terms in the chiral expansion which I expect to be the dominant source of uncertainty in our calculations.

Residual cutoff dependence of observables provides one possible way to estimate theoretical uncertainty due to truncation of the chiral expansion. However, as pointed out already in [48], such an approach suffers from several drawbacks: First, the residual cutoff dependence measures the impact of neglected contact interactions which contribute only at even orders $Q^{2 n}, n=0,1,2, \ldots$. This results in underestimation of uncertainties at NLO and $\mathrm{N}^{3} \mathrm{LO}$. Secondly, the available cutoff range is, in practice, rather limited, and attempts to increase the cutoff range by employing softer regulators are likely to cause an unnecessary increase of finite-cutoff artifacts. The "cutoff bands" can, therefore, generally not be expected to provide a reliable estimation of the theoretical uncertainty, see $[48,18]$ for more detail.

In order to circumvent these problems, we proposed in Ref. [18] a novel approach to error analysis by directly estimating the size of neglected higher-order terms. Let $X(p)$ be a given observable with $p$ referring to the corresponding momentum scale and $X^{(i)}(p), i=0,2,3, \ldots$, a prediction at order $Q^{i}$ in the chiral expansion. We further define the order- $Q^{i}$ corrections to $X(p)$ as

$$
\Delta X^{(2)} \equiv X^{(2)}-X^{(0)}, \quad \text { and } \quad \Delta X^{(i)} \equiv X^{(i)}-X^{(i-1)} \text { for } i \geq 3,
$$

so that the chiral expansion for $X$ up to order $Q^{i}$ takes the form

$$
X^{(i)}=X^{(0)}+\Delta X^{(2)}+\ldots+\Delta X^{(i)}, \text { with } \quad \Delta X^{(i)}=\mathscr{O}\left(Q^{i} X^{(0)}\right) .
$$

One can now use the information on $X^{(0)}$ and $\Delta X^{(j)}, j \leq i$, available upon performing explicit calculations, to quantify the theoretical uncertainty $\delta X^{(i)}$ of the order- $Q^{i}$ result by estimating the size of neglected higher-order terms. Specifically, the following procedure was employed in Refs. [18, 19]:

$$
\delta X^{(0)}=Q^{2}\left|X^{(0)}\right|, \quad \delta X^{(i)}=\max _{2 \leq j \leq i}\left(Q^{i+1}\left|X^{(0)}\right|, Q^{i+1-j}\left|\Delta X^{(j)}\right|\right) \text { with } i \geq 2,
$$




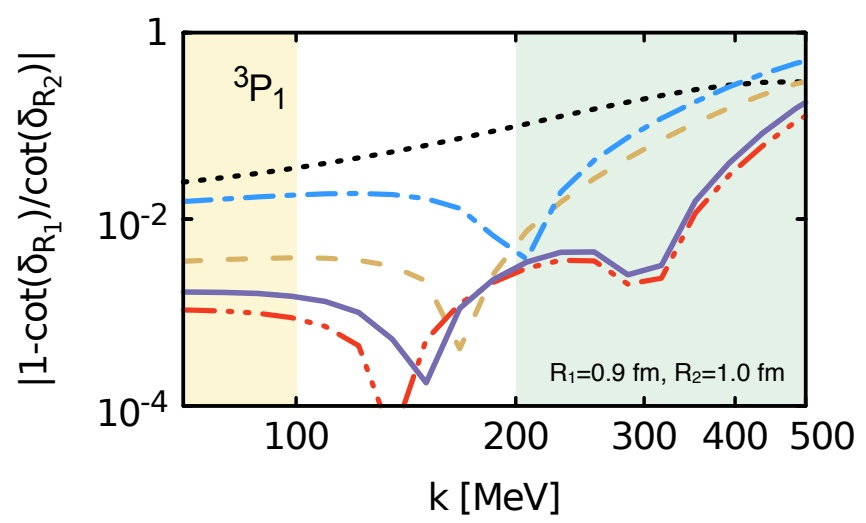

Figure 5: Error plot for $\mathrm{np}^{3} \mathrm{P}_{1}$ phase shift. Black dotted, brown dashed, blue dashed-dotted, red dashed-double-dotted and violet solid lines show the results at LO, NLO, $\mathrm{N}^{2} \mathrm{LO}, \mathrm{N}^{3} \mathrm{LO}$ and $\mathrm{N}^{4} \mathrm{LO}$, respectively. The light-shaded yellow (green) area shows the range of momenta where the error is expected to be dominated by neglected $M_{\pi} / \Lambda_{b}$-contributions ( $p / \Lambda_{b}$-contributions).

where the expansion parameter $Q$ was chosen as $Q=\max \left(p / \Lambda_{b}, M_{\pi} / \Lambda_{b}\right)$, subject to the additional constraint

$$
\delta X^{(i)} \geq \max _{j, k}\left(\left|X^{(j \geq i)}-X^{(k \geq i)}\right|\right) .
$$

The breakdown scale $\Lambda_{b}$ of the chiral expansion can be estimated e.g. from error plots such as the ones proposed by Lepage [70] or by Grießhammer, see [89] for more detail. An example of the latter one for the ${ }^{3} \mathrm{P}_{1}$ np partial wave is shown in Fig. 5. The depicted error function $\left|1-\cot \delta_{R_{1}}(k) / \cot \delta_{R_{2}}(k)\right|$ with $k$ referring to the CMS momentum measures the residual cutoff dependence of the phase shift. The observed flat behavior of the error function for $k$ well below $M_{\pi}$ (yellow-shaded area) is in line with the expectation that the error in this regime is dominated by neglected $M_{\pi} / \Lambda_{b}$-terms. On the other hand, the uncertainty at large momenta (green-shaded area) is dominated by neglected $k / \Lambda_{b}$-terms, and one indeed observes an increased slope at $\mathrm{N}^{3} \mathrm{LO} / \mathrm{N}^{4} \mathrm{LO}$ versus NLO/N ${ }^{2} \mathrm{LO}$ versus LO. ${ }^{6}$ The observed nearly identical slopes at NLO and $\mathrm{N}^{2} \mathrm{LO}$ as well as $\mathrm{N}^{3} \mathrm{LO}$ and $\mathrm{N}^{4} \mathrm{LO}$ reflect the same number of contact interactions at those orders. The spikes in the plot emerge when $\delta_{R_{1}}=\delta_{R_{2}}$ for some value of $k$ and should be ignored. The error plots can be used to read off the breakdown scale $\Lambda_{b}$, which corresponds to momenta at which the different lines cross each other. For $\mathrm{N}^{3} \mathrm{LO}, \Lambda_{b}$ was estimated in Ref. [18] to be $\Lambda_{b}=600 \mathrm{MeV}$ for $R=0.8-1.0 \mathrm{fm}, \Lambda_{b}=500 \mathrm{MeV}$ for $R=1.1 \mathrm{fm}$ and $\Lambda_{b}=400 \mathrm{MeV}$ for $R=1.2 \mathrm{fm}$.

It should be understood that the proposed approach to uncertainty quantification assumes the validity of Eq. (3.2), which relies on naive dimensional analyis and does not explicitly account for near-threshold enhancement of the amplitude in the case of an unnaturally large scattering length. We, however, expect this to be largely accounted for implicitly through employing in the error analysis the information about the actual size of $\Delta X^{(i)}$.

\footnotetext{
${ }^{6}$ Interpretation of such error plots should, however, be taken with care. In particular, the dependence of the lines in a double-logarithmic plot deviates from the linear one in the presence of the long-range interaction due to exchange of $\operatorname{pion}(\mathrm{s})$.
} 

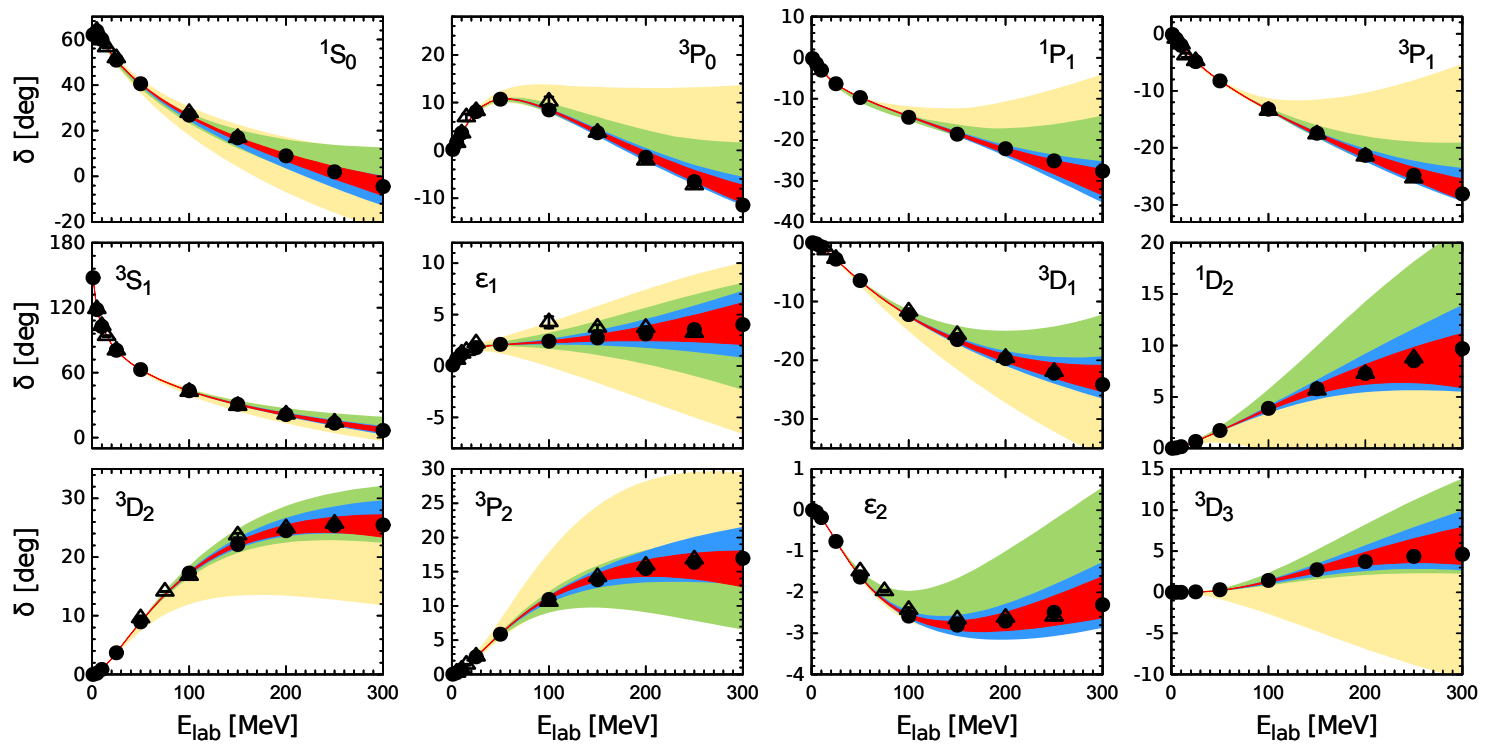

Figure 6: Results for the np S-, P- and D- waves and the mixing angles $\varepsilon_{1}, \varepsilon_{2}$ up to $\mathrm{N}^{4} \mathrm{LO}$ based on the cutoff of $R=0.9 \mathrm{fm}$ in comparison with the NPWA [84] (solid dots) and the GWU single-energy PWA [85] (open triangles). The shaded bands of increasing width show theoretical uncertainty at $\mathrm{N}^{4} \mathrm{LO}$ (red), $\mathrm{N}^{3} \mathrm{LO}$ (blue), $\mathrm{N}^{2} \mathrm{LO}$ (green) and NLO (yellow) estimated via Eqs. (3.3) and (3.4).

In the following, I will apply the approach for error analysis outlined above to selected observables in the NN system. I emphasize that our method does, of course, not require the knowledge of experimental data and is applicable to any observable of interest and for any particular choice of the regulator $R$ since it does not rely on cutoff variation.

Fig. 6 shows our results for phase shifts calculated up to $\mathrm{N}^{4} \mathrm{LO}$ for $R=0.9 \mathrm{fm}$ already depicted in Fig. 3, which are now furnished with the estimated theoretical uncertainties using Eqs. (3.3), (3.4). The various bands result by adding/subtracting the estimated theoretical uncertainty to/from the calculated results. Similarly, we show in Fig. 7 our predictions for the np total cross section at various energies using the same value of the regulator in comparison with the result of the NPWA. As in the case of phase shifts, one observes a very good convergence of the chiral expansion and excellent agreement between the theoretical predictions, NPWA and experimental data. The convergence appears to be very fast at the lowest considered energy and, as expected, slows down at $E_{\text {lab }}=200 \mathrm{MeV}$, where the $\mathrm{N}^{4} \mathrm{LO}$ predictions are, however, still accurate within a few percent. Our quoted theoretical uncertainties for the total cross section and the case of $R=0.9 \mathrm{fm}$ were found in [91] to be consistent with the $68 \%$ degree-of-belief intervals for EFT predictions. As another application, we show in Fig. 8 our predictions for a selected set of np scattering observables at $E_{\text {lab }}=143 \mathrm{MeV}$ based on $R=0.9 \mathrm{fm}$. In all cases, we observe excellent agreement with the NPWA and confirm a good convergence of the chiral expansion. More results for NN observables can be found in Refs. [18, 19].

As already advertised, the novel approach to uncertainty quantification is not restricted to a particular choice of the regulator. Carrying out the error analysis for calculations based on different choices of $R$ thus provides a useful consistency check of the method. In Fig. 9, we show the results 

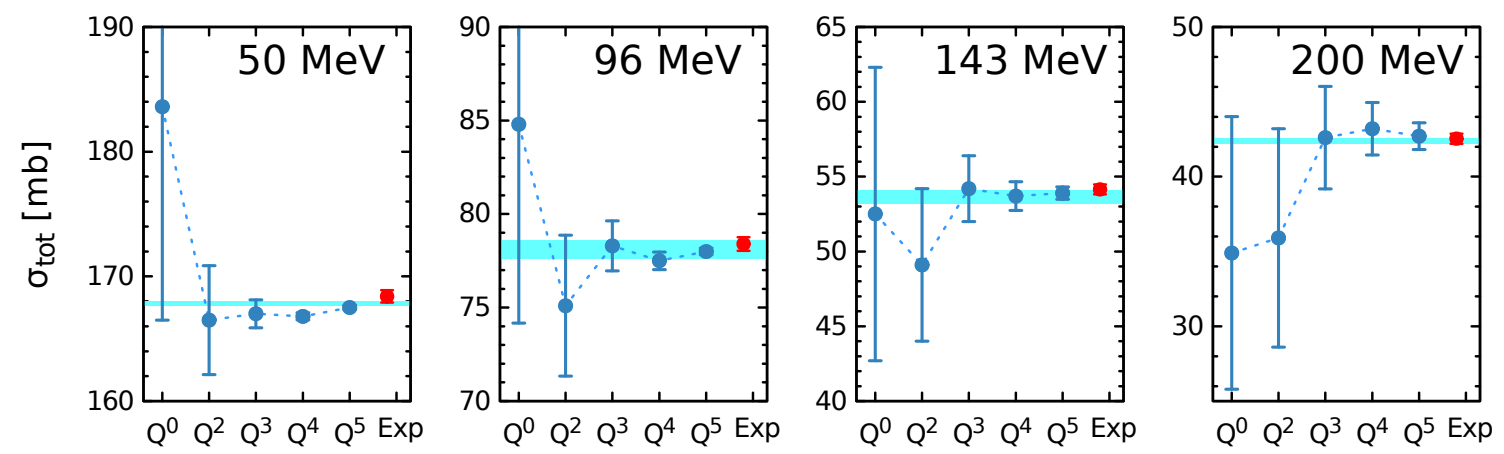

Figure 7: Chiral expansion of the np total cross section at different energies based on $R=0.9 \mathrm{fm}$ in comparison with experimental data of Ref. [90]. The horizontal band shows the result of the NPWA.

Table 2: Deuteron binding energy $B_{d}$ (in $\mathrm{MeV}$ ), asymptotic $S$ state normalization $A_{S}$ (in $\mathrm{fm}^{-1 / 2}$ ), asymptotic $D / S$ state ratio $\eta$, radius $r_{d}$ (in $\mathrm{fm}$ ) and quadrupole moment $Q$ (in $\mathrm{fm}^{2}$ ) based on the cutoff $R=0.9 \mathrm{fm}$ in comparison with empirical information. Also shown is the $D$-state probability $P_{D}$ (in \%). Notice that $r_{d}$ and $Q$ are calculated without taking into account exchange current contributions and relativistic corrections. References to experimental data can be found in Ref. [18].

\begin{tabular}{lllllll}
\hline & $\mathrm{LO}$ & $\mathrm{NLO}$ & $\mathrm{N}^{2} \mathrm{LO}$ & $\mathrm{N}^{3} \mathrm{LO}$ & $\mathrm{N}^{4} \mathrm{LO}$ & Empirical \\
\hline$B_{d}$ & 2.0235 & 2.1987 & 2.2311 & $2.2246^{\star}$ & $2.2246^{\star}$ & $2.224575(9)$ \\
$A_{S}$ & 0.8333 & 0.8772 & 0.8865 & 0.8845 & 0.8844 & $0.8846(9)$ \\
$\eta$ & 0.0212 & 0.0256 & 0.0256 & 0.0255 & 0.0255 & $0.0256(4)$ \\
$r_{d}$ & 1.990 & 1.968 & 1.966 & 1.972 & 1.972 & $1.97535(85)$ \\
$Q$ & 0.230 & 0.273 & 0.270 & 0.271 & 0.271 & $0.2859(3)$ \\
$P_{D}$ & 2.54 & 4.73 & 4.50 & 4.19 & 4.29 & \\
\hline
\end{tabular}

${ }^{\star}$ The deuteron binding energy has been taken as input in the fit.

for the total cross section at all orders starting from NLO and for all considered cutoff choices. Within the quoted errors, the predictions based on different values of $R$ agree with each other and the NPWA for all orders in the chiral expansion. The accuracy of the predicted results for the cross section shows the same dependence on the cutoff as the quality of the fits discussed in section 2.4.

In Table 2, we list our results for the deuteron properties. At the considered accuracy level, the chiral expansion is nearly converged already at $\mathrm{N}^{3} \mathrm{LO}$ except for $P_{D}$ which is not an observable quantity. ${ }^{7}$ The predicted values for $A_{S}$ and $\eta$ are in excellent agreement with the empirical numbers. Using Eqs. (3.3) and (3.4) and adopting $Q=M_{\pi} / \Lambda_{b}$, our predictions for $A_{S}$ at $\mathrm{N}^{4} \mathrm{LO}$ is $A_{S}=$ $0.8844 \pm 0.0002 \mathrm{fm}^{-1 / 2}$ while the accuracy for $\eta$ is beyond the quoted figures. For the $r_{d}$ and $Q$, our results are incomplete as we do not include relativistic corrections and meson-exchange current contributions. The estimated size of these corrections is consistent with the deviation between our

\footnotetext{
${ }^{7} P_{D}=5 \% \pm 1 \%$ has been used as an additional "data" point in the fits at $\mathrm{N}^{3} \mathrm{LO}$ and $\mathrm{N}^{4} \mathrm{LO}$ in order to stabilize the results, see Ref. [18] for more detail.
} 

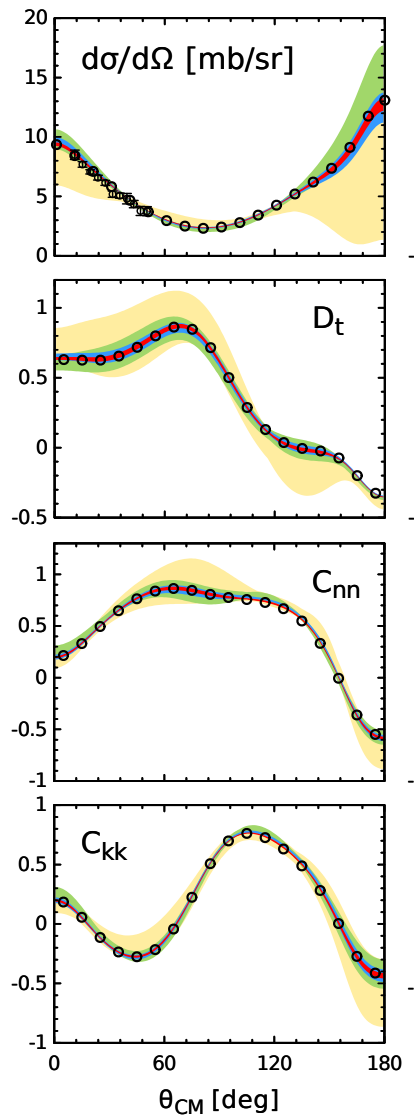
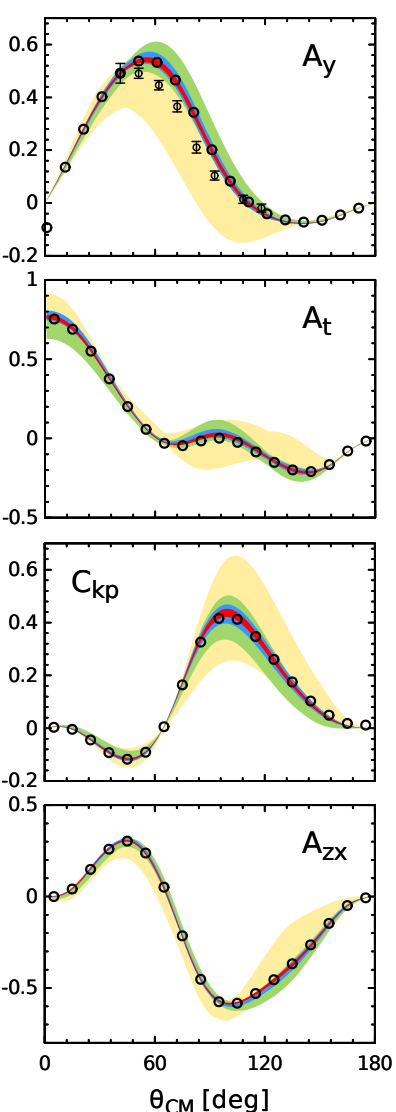
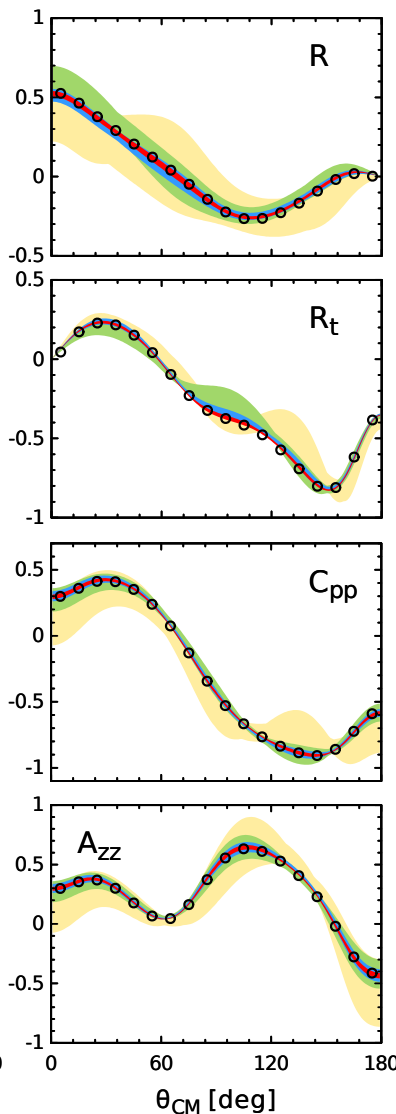

Figure 8: Predictions for the np differential cross section $d \sigma / d \Omega$, the analyzing power $A_{y}$, the rotation parameter $R$, the polarization-transfer parameters $D_{t}, R_{t}$ and $A_{t}$ and the spin-correlation parameters $C_{n n}, C_{k p}, C_{p p}, C_{k k}, A_{z x}$ and $A_{z z}$ at $E_{\text {lab }}=143 \mathrm{MeV}$ calculated up to $\mathrm{N}^{4} \mathrm{LO}$ based on the cutoff of $R=0.9 \mathrm{fm}$. Data for the cross section are at $E_{\mathrm{lab}}=142.8 \mathrm{MeV}$ and taken from [92] and for the analyzing power from [93]. For remaining notation see Fig. 6.

values and the empirical numbers, see [18] for an extended discussion.

\section{Beyond the two-nucleon system}

Having developed the new generation of $\mathrm{NN}$ potentials up to $\mathrm{N}^{4} \mathrm{LO}$ and the novel approach to uncertainty quantification, which has been validated in the NN system, we are well prepared to test nuclear chiral EFT in heavier systems and to systematically analyze the role of the 3NF, which has been the subject of intense experimental research at FZ Jülich, GANIL, KVI, RIKEN, TUNL and other laboratories. This is the main goal of the recently formed Low Energy Nuclear Physics International Collaboration (LENPIC). The numerical implementation of the $3 \mathrm{NF}$ regularized in the same way as the NN potentials of Refs. $[18,19]$ is currently in progress so that no results including the novel NN potentials and 3NF are available at this stage. Still, as argued in [94], it is instructive to analyze few-nucleon systems based on NN forces only. Consider, for example, the nucleon-deuteron $(\mathrm{Nd})$ total cross section. Our predictions for this observable at four different 

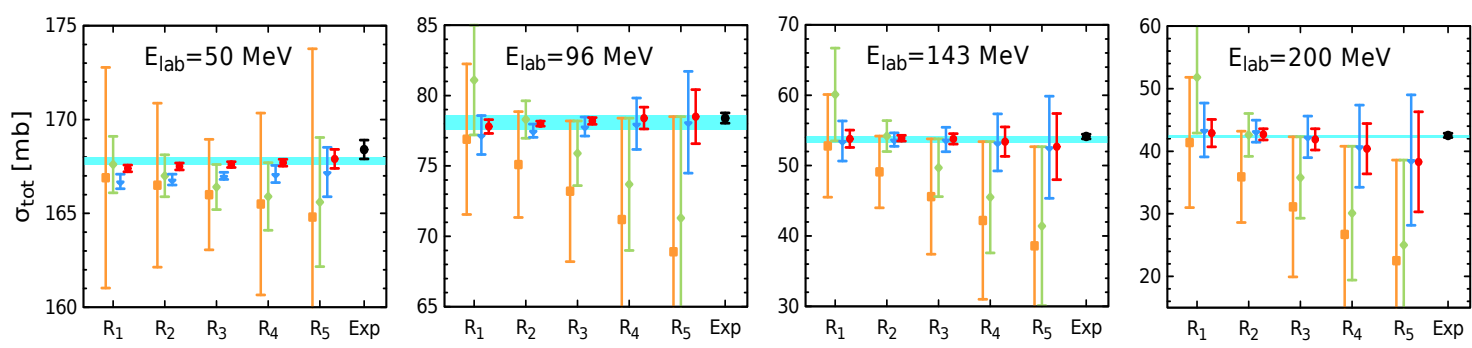

Figure 9: Predictions for the np total cross section at NLO (filled orange squares), $\mathrm{N}^{2} \mathrm{LO}$ (solid green diamonds), $\mathrm{N}^{3} \mathrm{LO}$ (filled blue triangles) and $\mathrm{N}^{4} \mathrm{LO}$ (filled red circles) for different choices of the cutoff, see Eq. (2.8) in comparison with NPWA (the horizontal bands) and data of Ref. [90].
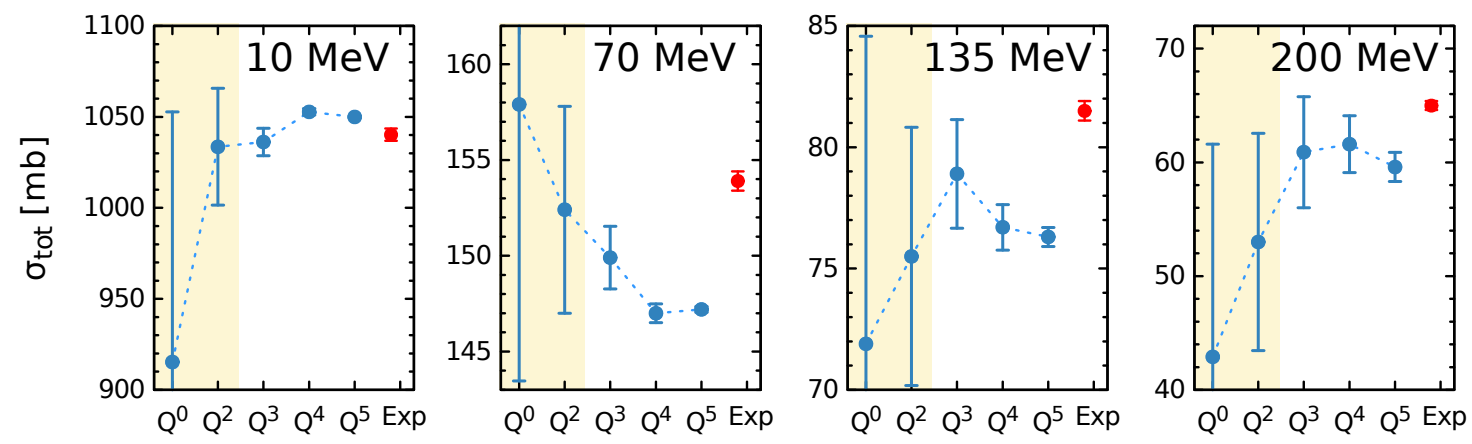

Figure 10: Chiral expansion of the $\mathrm{Nd}$ total cross section at different energies based on the cutoff or $R=0.9 \mathrm{fm}$ and using $\mathrm{NN}$ forces only in comparison with experimental data of Ref. [90].

energies are summarized in Fig. 10 for the case of $R=0.9 \mathrm{fm}$. The light-shaded yellow area in the plots indicates that our results are complete only up to NLO due to missing $3 \mathrm{NF}$. One observes a significant discrepancy between the theoretical predictions based on the NN forces only and data, which provides clear evidence for missing 3NF contributions, cf. Figs. 7 and 10. The estimated accuracy of the chiral EFT results at $\mathrm{N}^{4} \mathrm{LO}$ suggests that $\mathrm{Nd}$ scattering at intermediate energies will be a very promising testing ground for the chiral 3NF. Furthermore, it is comforting to see that the deviations between our $\mathrm{N}^{3} \mathrm{LO} / \mathrm{N}^{4} \mathrm{LO}$ predictions and experimental data are similar in size to the NLO error bars, which give the estimated size of $\mathrm{N}^{2} \mathrm{LO}$ contributions. This is precisely the chiral order at which the first nonvanishing $3 \mathrm{NF}$ appears according to Weinberg's power counting. Our results do not indicate the need to promote the short-range 3NF operators to lower orders as suggested e.g. in Ref. [83]. For more examples of few-nucleon observables see Ref. [94].

\section{Summary and outlook}

In this talk I presented the new generation of NN potentials derived in chiral EFT up to $\mathrm{N}^{4} \mathrm{LO}$. We were able to significantly reduce finite-cutoff artifacts by using an appropriate regularization in coordinate space which maintains the analytic structure of the amplitude. The new potentials do not require the additional spectral function regularization and employ the LECs $c_{i}, d_{i}$ and $e_{i}$ determined from $\pi N$ scattering without any fine tuning. We found a clear evidence of the corresponding parameter-free two-pion exchange contributions by observing an improved description of 
NN phase shifts at $\mathrm{N}^{2} \mathrm{LO}$ and $\mathrm{N}^{4} \mathrm{LO}$. Furthermore, a simple approach for estimating the theoretical uncertainty in few-nucleon calculations from the truncation of the chiral expansion, that does not rely on cutoff variation, was formulated and validated in the NN system.

Our work opens up new perspectives for precision ab initio calculations in few- and manynucleon systems and is especially relevant for ongoing efforts towards a quantitative understanding of the structure of the 3NF in the framework of chiral EFT, see [94] for a first step along this line.

\section{References}

[1] S. Weinberg, Phys. Lett. B 251, 288 (1990).

[2] S. Weinberg, Nucl. Phys. B 363, 3 (1991).

[3] E. Epelbaum, H. W. Hammer and U.-G. Meißner, Rev. Mod. Phys. 81, 1773 (2009).

[4] R. Machleidt and D. R. Entem, Phys. Rept. 503, 1 (2011).

[5] N. Kalantar-Nayestanaki et al., Rept. Prog. Phys. 75, 016301 (2012).

[6] H. W. Hammer, A. Nogga and A. Schwenk, Rev. Mod. Phys. 85, 197 (2013).

[7] G. Hagen et al., Phys. Rev. Lett. 108, 242501 (2012).

[8] E. D. Jurgenson et al., Phys. Rev. C 87, no. 5, 054312 (2013).

[9] A. Lovato et al., Phys. Rev. Lett. 111, no. 9, 092501 (2013).

[10] H. Hergert et al., Phys. Rev. C 87, no. 3, 034307 (2013).

[11] V. Soma, C. Barbieri, T. Duguet, Phys. Rev. C 87, no. 1, 011303 (2013).

[12] D. Lee, Prog. Part. Nucl. Phys. 63, 117 (2009).

[13] E. Epelbaum et al., Phys. Rev. Lett. 106, 192501 (2011).

[14] E. Epelbaum et al., Phys. Rev. Lett. 109, 252501 (2012).

[15] E. Epelbaum et al., Phys. Rev. Lett. 112, no. 10, 102501 (2014).

[16] S. Elhatisari et al., arXiv:1506.03513 [nucl-th].

[17] Ulf-G. Meißner, these proceedings.

[18] E. Epelbaum, H. Krebs and U.-G. Meißner, Eur. Phys. J. A 51, no. 5, 53 (2015).

[19] E. Epelbaum, H. Krebs and U.-G. Meißner, Phys. Rev. Lett. 115, no. 12, 122301 (2015).

[20] N. Fettes et al., Annals Phys. 283, 273 (2000) [Annals Phys. 288, 249 (2001)].

[21] S. Beane, these proceedings.

[22] M. Schindler, these proceedings.

[23] T. R. Hemmert, B. R. Holstein and J. Kambor, J. Phys. G 24, 1831 (1998).

[24] E. Epelbaum, Eur. Phys. J. A 34, 197 (2007).

[25] S. Weinberg, Phys. Lett. B 295, 114 (1992).

[26] U. van Kolck, Phys. Rev. C 49, 2932 (1994).

[27] C. Ordonez, L. Ray and U. van Kolck, Phys. Rev. C 53, 2086 (1996). 
[28] S. Pastore et al., Phys. Rev. C 80, 034004 (2009).

[29] S. Pastore et al., Phys. Rev. C 84, 024001 (2011).

[30] M. Piarulli et al., Phys. Rev. C 87, no. 1, 014006 (2013).

[31] A. Baroni et al., arXiv:1509.07039 [nucl-th].

[32] R. Schiavilla, these proceedings.

[33] M. Taketani, S. Machida and S. Onuma, Prog. Theore. Phys. 7 (1952) 45.

[34] S. Okubo, Prog. Theor. Phys. 12, 603 (1954).

[35] E. Epelbaum, W. Glöckle and U.-G. Meißner, Nucl. Phys. A 637, 107 (1998).

[36] E. Epelbaum, U.-G. Meißner and W. Glöckle, Nucl. Phys. A 714, 535 (2003).

[37] E. Epelbaum, Prog. Part. Nucl. Phys. 57, 654 (2006).

[38] H. Krebs, V. Bernard and U.-G. Meißner, Annals Phys. 316, 160 (2005).

[39] S. Kölling et al., Phys. Rev. C 80, 045502 (2009).

[40] S. Kölling et al., Phys. Rev. C 84, 054008 (2011).

[41] N. Kaiser, R. Brockmann and W. Weise, Nucl. Phys. A 625, 758 (1997).

[42] E. Epelbaum, Phys. Lett. B 639, 456 (2006).

[43] N. Kaiser, Phys. Rev. C 61, 014003 (2000).

[44] N. Kaiser, Phys. Rev. C 62, 024001 (2000).

[45] N. Kaiser, Phys. Rev. C 64, 057001 (2001).

[46] N. Kaiser, Phys. Rev. C 65, 017001 (2002).

[47] D. R. Entem and R. Machleidt, Phys. Rev. C 68, 041001 (2003).

[48] E. Epelbaum, W. Glöckle and U.-G. Meißner, Nucl. Phys. A 747, 362 (2005).

[49] D. R. Entem et al., Phys. Rev. C 91, no. 1, 014002 (2015).

[50] D. R. Entem et al., arXiv:1505.03562 [nucl-th].

[51] E. Epelbaum et al., Phys. Rev. C 66, 064001 (2002).

[52] S. Ishikawa and M. R. Robilotta, Phys. Rev. C 76, 014006 (2007).

[53] V. Bernard et al., Phys. Rev. C 77, 064004 (2008).

[54] V. Bernard et al., Phys. Rev. C 84, 054001 (2011).

[55] H. Krebs, A. Gasparyan and E. Epelbaum, Phys. Rev. C 85, 054006 (2012).

[56] H. Krebs, A. Gasparyan and E. Epelbaum, Phys. Rev. C 87, no. 5, 054007 (2013).

[57] L. Girlanda, A. Kievsky and M. Viviani, Phys. Rev. C 84, 014001 (2011).

[58] H. Krebs, these proceedings.

[59] D. B. Kaplan, M. J. Savage and M. B. Wise, Nucl. Phys. B 534, 329 (1998).

[60] S. Fleming, T. Mehen and I. W. Stewart, Nucl. Phys. A 677, 313 (2000).

[61] E. Epelbaum and J. Gegelia, Phys. Lett. B 716, 338 (2012). 
[62] V. G. Kadyshevsky, Nucl. Phys. B 6, 125 (1968).

[63] E. Epelbaum and J. Gegelia, PoS CD 12, 090 (2013).

[64] E. Epelbaum et al., Eur. Phys. J. A 50, 51 (2014).

[65] E. Epelbaum et al., Eur. Phys. J. A 51, no. 6, 71 (2015).

[66] A. Nogga, R. G. E. Timmermans and U. van Kolck, Phys. Rev. C 72, 054006 (2005).

[67] M. Pavon Valderrama and E. Ruiz Arriola, Phys. Rev. C 74, 054001 (2006).

[68] S. R. Beane et al., Nucl. Phys. A 700, 377 (2002).

[69] E. Epelbaum and J. Gegelia, Eur. Phys. J. A 41, 341 (2009).

[70] G. P. Lepage, nucl-th/9706029.

[71] E. Epelbaum and U.-G. Meißner, Few Body Syst. 54, 2175 (2013).

[72] V. Baru et al., Eur. Phys. J. A 48, 69 (2012).

[73] A. Gezerlis et al., Phys. Rev. Lett. 111, no. 3, 032501 (2013).

[74] M. Piarulli et al., Phys. Rev. C 91, no. 2, 024003 (2015).

[75] E. Epelbaum, W. Glöckle and U.-G. Meißner, Eur. Phys. J. A 19, 125 (2004).

[76] E. Marji et al., Phys. Rev. C 88, no. 5, 054002 (2013).

[77] N. Fettes, U.-G. Meißner and S. Steininger, Nucl. Phys. A 640, 199 (1998).

[78] P. Büttiker and U.-G. Meißner, Nucl. Phys. A 668, 97 (2000).

[79] K. A. Wendt, B. D. Carlsson and A. Ekström, arXiv:1410.0646 [nucl-th].

[80] M. Hoferichter et al., arXiv:1507.07552 [nucl-th], to appear in Phys. Rev. Lett..

[81] M. Hoferichter et al., JHEP 1206, 063 (2012).

[82] B. Long and C. J. Yang, Phys. Rev. C 85, 034002 (2012).

[83] M. C. Birse, Phil. Trans. Roy. Soc. Lond. A 369, 2662 (2011).

[84] V. G. J. Stoks et al., Phys. Rev. C 48, 792 (1993).

[85] R. A. Arndt, I. I. Strakovsky and R. L. Workman, Phys. Rev. C 50, 2731 (1994).

[86] V. G. J. Stoks et al., Phys. Rev. C 49, 2950 (1994).

[87] R. J. Furnstahl, D. R. Phillips and S. Wesolowski, J. Phys. G 42, no. 3, 034028 (2015).

[88] A. Ekström et al., J. Phys. G 42, no. 3, 034003 (2015).

[89] H. Grießhammer, these proceedings.

[90] W. P. Abfalterer et al., Phys. Rev. C 63, 044608 (2001).

[91] R. J. Furnstahl et al., Phys. Rev. C 92, no. 2, 024005 (2015).

[92] A.J. Bersbach et al., Phys. Rev. D 13 (1976) 535.

[93] A.F. Kuckes et al., Phys. Rev. 121 (1961) 1226.

[94] S. Binder et al., arXiv:1505.07218 [nucl-th]. 